\title{
A concise review of magnetic resonance molecular imaging of tumor angiogenesis by targeting integrin $\alpha v \beta 3$ with magnetic probes
}

This article was published in the following Dove Press journal:

International Journal of Nanomedicine

13 March 2013

Number of times this article has been viewed

Yajie Liu

Yi Yang

Chunfu Zhang

School of Biomedical Engineering and Med-X Research Institute,

Shanghai Jiao Tong University,

Shanghai, People's Republic of China
Correspondence: Chunfu Zhang School of Biomedical Engineering and Med-X Research Institute, Shanghai Jiao Tong University,

1954 Huashan Road, Shanghai 200030, People's Republic of China Tel +862162933323

Fax +862162932907

Email cfzhang@sjtu.edu.cn
Abstract: Angiogenesis is an essential step for the growth and spread of malignant tumors. Accurate detection and quantification of tumor angiogenesis is important for early diagnosis of cancers as well as post therapy assessment of antiangiogenic drugs. The cell adhesion molecule integrin $\alpha v \beta 3$ is a specific marker of angiogenesis, which is highly expressed on activated and proliferating endothelial cells, but generally not on quiescent endothelial cells. Therefore, in recent years, many different approaches have been developed for imaging $\alpha v \beta 3$ expression, for the detection and characterization of tumor angiogenesis. The present review provides an overview of the current status of magnetic resonance molecular imaging of integrin $\alpha v \beta 3$, including the new development of high sensitive contrast agents and strategies for improving the specificity of targeting probes and the biological effects of imaging probes on $\alpha v \beta 3$ positive cells.

Keywords: integrin $\alpha v \beta 3$, molecular imaging, MRI, paramagnetic liposome, superparamagnetic iron oxide particles, tumor angiogenesis

\section{Introduction to tumor angiogenesis}

Angiogenesis is the process by which new blood vessels arise from existing ones. Physiologically, angiogenesis is present in adults during wound healing, the development of the ovarian follicle and corpus luteum, and in the proliferating endometrium. ${ }^{1}$ Pathological angiogenesis is also a component of much diverse pathologies ranging from diabetes and atherosclerosis to cancer, a disease that cannot progress without the formation of new blood vessels. ${ }^{2}$ Tumors in particular rely on angiogenesis for their continued growth. ${ }^{3,4}$ Solid tumors will not grow larger than $2-3 \mathrm{~mm}$ in diameter in the absence of new blood vessels. ${ }^{5}$ When tumors grow beyond this size, the increased interstitial pressure within the tumor inhibits the diffusion of metabolites and nutrients necessary for tumor growth and a state of cellular hypoxia begins. Hypoxia increases cellular hypoxia-inducible factor transcription. Binding of hypoxia-inducible factor to the hypoxia response element turns on the expression of vascular endothelial growth factor, a key player in the angiogenic process. In addition to vascular endothelial growth factor, a variety of regulating factors (eg, transforming growth factor- $\beta$, platelet-derived growth factor, basic fibroblast growth factor, and acidic fibroblast growth factor) are involved in this process, which are locally secreted by numerous cells, such as stromal, endothelial cells, and cancer cells, but can also derive from blood and the extracellular matrix. ${ }^{6,7}$ After activation of endothelial cells, proteolytic enzymes, such as serine proteases and matrix metalloproteinases, are excreted, allowing degradation of the basement membrane and the extracellular matrix surrounding the vessels. ${ }^{8}$ After this degradation, endothelial cells, mediated by cell adhesion receptors, like the integrin 
$\alpha v \beta 3$, proliferate and migrate until they form unstable microvessels. In a further step, mesenchymal cells release angiopoietin-1, which interacts with Tie-2 receptor tyrosine kinase mediating capillary organization and stabilization. ${ }^{9}$ The newly built endothelial cells form tight junctions with each other, leading to new tubes that are connected with the microcirculation. Blood flow can then be established. ${ }^{10}$ The detailed mechanisms of angiogenesis are described in previous reports. ${ }^{1,11}$

Various classes of adhesion molecules are involved in tumor angiogenesis. Members of the integrin, cadherin, selectin, and immunoglobulin families contribute to each step of tumor vascularization, not only by mediating cellcell and cell-matrix interactions, but also by participating in those signaling events that regulate the extension and the maturation of neoforming vessels. ${ }^{12,13}$ These molecules have been exploited as targets for molecular imaging and quantification of tumor angiogenesis. ${ }^{14}$ Among these molecules, integrins have been extensively studied. ${ }^{15}$ Integrins are heterodimeric transmembrane glycoproteins, which consist of two noncovalently bound transmembrane subunits with large extracellular segments that bind to create heterodimers with distinct adhesive capabilities. ${ }^{16} \mathrm{Up}$ to now, $18 \alpha$ and eight $\beta$ subunits have been described, which assemble into 24 different integrins (receptors). Among all 24 integrins discovered to date, integrin $\alpha v \beta 3$ is the most extensively studied, ${ }^{17-19}$ which is significantly upregulated on activated endothelial cells during angiogenesis, but not on quiescent endothelial cells. ${ }^{20}$ Clinical studies showed that the expression of integrin $\alpha v \beta 3$ correlated with tumor grade, ${ }^{21,22}$ and thus suggested integrin $\alpha v \beta 3$ as a marker of malignancy. Therefore, the ability to noninvasively detect $\alpha v \beta 3$ expression in living subjects would allow a better characterization of tumors and help to identify tumor regions with higher aggressiveness. In this context, in the past few years, a vast array of sensitive molecular imaging agents associated with angiogenesisspecific targets have been developed for molecular imaging of integrin $\alpha v \beta 3$ expression for early detection of cancers using different modalities. ${ }^{19,23,24}$

\section{Modalities used for tumor angiogenesis imaging}

The modalities that have been proposed for imaging tumor angiogenesis include magnetic resonance imaging (MRI), positron emission tomography (PET), single photon emission-computed tomography (SPECT), ultrasound, and optical imaging. ${ }^{15,25}$ These systems provide a wealth of information that is highly complementary. PET and SPECT are very sensitive and highly quantitative, have no limitation of penetration depth, but both involve the use of ionizing radiation, which is impractical for frequent imaging due to potential damage to the living subjects from the cumulative irradiation dose. Optical imaging is also very sensitive; however, it lacks depth penetration in tissue (ie, limited to surface imaging; for example, skin cancer, esophagus or colon cancer with optical endoscopy, or bladder cancer imaging with optical cystoscopy). ${ }^{26,27}$ Thus, its applications are also limited. Moreover, PET, SPECT, and optical imaging have low spatial resolution. Ultrasound imaging is inexpensive, widely available and completely noninvasive; therefore, it is a promising technique for the evaluation of tumor angiogenesis. However, the limitations of ultrasound are the dependence on the skill of the operator and limited depth penetration. Moreover, not all regions of the body are accessible with ultrasound (lung, bone, and brain in adults), and adequate documentation for comparison of examinations at different time points is still problematic in a clinical setting. ${ }^{28}$

MRI is widely used clinically to assess tumor growth and for response evaluation, which generally measures the magnetic relaxation properties of protons, but this requires excitation by radiofrequency. Radiofrequency waves have wavelengths in the order of $1 \mathrm{~cm}$ and can penetrate deep into the body. Therefore, MRI has no limitation for tissue penetration. ${ }^{29}$ Moreover, compared to PET and SPECT, MRI does not use ionizing radiation and offers higher resolution and soft tissue contrast. In addition, MRI allows for multiparametric imaging in which $\mathrm{T} 1, \mathrm{~T} 2$, and diffusion-weighted images (DWI) are obtained in one session, each reflecting a different tissue signal. ${ }^{30}$ Clinically, multiparametric imaging combining DWI with anatomical T1-weighted imaging dramatically increased specificity and accuracy of bone metastases detection in patients with lung cancer compared to DWI alone. ${ }^{31}$ Similarly, the combination of DWI/T2-weighted imaging dramatically improved the sensitivity and specificity of DWI in the detection of abdominal malignancies including prostate cancer. ${ }^{30,32}$

Recent advances in whole-body diffusion-weighted imaging show promise for diagnosing lesions throughout the entire human body as well as for assessing lymph node metastases, with superior spatial resolution, and sensitivity and specificity. ${ }^{30}$ Furthermore, magnetic resonance (MR) spectroscopy can be used to assess tumor $\mathrm{pH}$ and metabolic activity. ${ }^{33}$ When combined with MRI, the functional and molecular information can be coregistered with anatomical information within a single imaging method, which would improve the diagnostic specificity of malignant cancers. 
These advantages make MRI highly desirable for molecular imaging. However, the sensitivity for detection of targeting agents is relatively low. Therefore, high sensitivity and specificity of the imaging probes for the targets are often necessary for successful detection of tumor angiogenesis. The comparison of different noninvasive imaging modalities is shown in Table 1.

\section{MR molecular imaging of tumor angiogenesis by targeting integrin $\alpha v \beta 3$ Imaging with gadolinium (Gd[III])- containing lipid nanoagents}

By far, the most widely used MRI contrast agents are those based on the paramagnetic gadolinium ion. The Gd(III) ion is chelated to low molecular weight ligands, such as diethylene triamine pentacetate acid (DTPA) and 1,4,7,10-Tetraazacyclododecane-1,4,7,10-tetraacetic acid to reduce toxicity. ${ }^{34}$ However, binding of few gadolinium complexes to angiogenic targets does not usually provide a sufficiently high signalto-noise ratio. In order to enhance the detection sensitivity, Gd(III) complexes are very often loaded onto nanocarriers. In this way, initial efforts to specifically visualize angiogenic targets were successful. The first MRI approach for imaging $\alpha v \beta 3$ expression on tumor angiogenesis was demonstrated by Sipkins et al. ${ }^{35}$ In this seminal study, cross-linked liposomes with a high payload of gadolinium (containing $30 \%$ of $\mathrm{Gd}[\mathrm{III}]$ chelate-labeled lipid) and a mean size of $300-350 \mathrm{~nm}$ were conjugated with an antiintegrin $\alpha v \beta 3$ antibody. After intravenous administration into rabbits with squamous cell carcinoma, the "hot spots" of tumor angiogenesis were able to be detected by MRI. Moreover, MR signal intensity enhancement correlated well with integrin $\alpha v \beta 3$ expression levels. Lanza et $\mathrm{al}^{36}$ have developed a highly MRI sensitive nanosystem that has a perfluorocarbon core covered with a monolayer of lipids. Paramagnetic lipids (eg, gadolinium-diethylenetriaminepentaacetic acid-bis-oleate) were incorporated into the lipid monolayer for detection with MRI. After conjugation with a peptidomimetic $\alpha v \beta 3$ antagonist, the paramagnetic perfluorocarbon nanoparticles were tried for tumor angiogenesis detection with a clinical 1.5 T MRI scanner. ${ }^{37}$ Dynamic imaging indicated that MR signal enhancement continued to build over at least 2 hours after probe injection, while the background blood pool effects were nondetectable. Despite the relatively large diameter of approximate $270 \mathrm{~nm}$, the probes penetrated into the leaky tumor neovasculature, but did not migrate substantially into the interstitium. In a following study, the same group extended these results and demonstrated that nude mice with human melanoma tumor xenografts with sizes of about $30 \mathrm{~mm}^{3}$ could also be detected with a clinical $1.5 \mathrm{~T}$ MRI scanner using the same integrin $\alpha v \beta 3$-targeted paramagnetic nanoparticles, ${ }^{38}$ which may enable characterizing and staging of early melanoma in a clinical setting.

Other endeavors for improving MR sensitivity for tumor angiogenesis imaging include developing Gd(III)-containing micelles, liposomes (a spherical vesicle composed of a bilayer of naturally occurring phospholipids), or high-density lipoprotein-like nanoparticles. ${ }^{39}$ These nano constructs can enable the detection in the picomolar range (particle concentration), and therefore enable visualization with MRI of sparse binding sites. ${ }^{40}$ Strijkers et $\mathrm{al}^{41}$ introduced a paramagnetic liposome, which consisted of disaturated-phosphatidylcholine (DSPE), poly(ethylene glycol) (PEG)2000-DSPE, maleimide-PEG2000-DSPE, cholesterol, and $25 \mathrm{~mol} \%$

Table I Characteristics of noninvasive imaging modalities ${ }^{71,72}$

\begin{tabular}{|c|c|c|c|c|c|}
\hline $\begin{array}{l}\text { Imaging } \\
\text { technique }\end{array}$ & $\begin{array}{l}\text { Positron emission } \\
\text { tomography }\end{array}$ & $\begin{array}{l}\text { Single photon emission } \\
\text { computed tomography }\end{array}$ & $\begin{array}{l}\text { Magnetic resonance } \\
\text { imaging }\end{array}$ & Ultrasound & $\begin{array}{l}\text { Optical } \\
\text { imaging }\end{array}$ \\
\hline Detection & $\begin{array}{l}\text { High energy } \\
\gamma \text { rays }\end{array}$ & $\begin{array}{l}\text { Lower energy } \\
\gamma \text { rays }\end{array}$ & Radio waves & $\begin{array}{l}\text { High frequency } \\
\text { sound }\end{array}$ & $\begin{array}{l}\text { Visible light } \\
\text { and infrared }\end{array}$ \\
\hline Resolution & $\mathrm{I}-2 \mathrm{~mm}$ & $\mathrm{I}-2 \mathrm{~mm}$ & $10-100 \mu \mathrm{m}$ & $50 \mu \mathrm{m}$ & $2-5 \mathrm{~mm}$ \\
\hline Depth & No limit & No limit & No limit & $\mathrm{mm}-\mathrm{cm}$ & $\mathrm{I}-2 \mathrm{~cm}$ \\
\hline Time & Minutes to hours & Minutes to hours & Minutes to hours & $\begin{array}{l}\text { Seconds to } \\
\text { minutes }\end{array}$ & $\begin{array}{l}\text { Seconds to } \\
\text { minutes }\end{array}$ \\
\hline Sensitivity & $10^{-11}-10^{-12} \mathrm{M}$ & $10^{-10}-10^{-11} \mathrm{M}$ & $10^{-3}-10^{-5} \mathrm{M}$ & $\begin{array}{l}\text { Not well } \\
\text { characterized }\end{array}$ & $10^{-9}-10^{-12} \mathrm{M}$ \\
\hline Imaging agents & $\begin{array}{l}{ }^{18} \mathrm{~F}-,{ }^{64} \mathrm{Cu}-,{ }^{11} \mathrm{C} \text {-labelled } \\
\text { compounds }\end{array}$ & $\begin{array}{l}{ }^{99 m} T c-\text { or } 11 ' I n \text {-labelled } \\
\text { compounds }\end{array}$ & $\begin{array}{l}\text { Paramagnetic chelates, } \\
\text { magnetic particles }\end{array}$ & Microbubbles & Fluorophores \\
\hline Target & $\begin{array}{l}\text { Physiological, } \\
\text { molecular }\end{array}$ & $\begin{array}{l}\text { Physiological, } \\
\text { molecular }\end{array}$ & $\begin{array}{l}\text { Anatomical, physiological, } \\
\text { molecular }\end{array}$ & $\begin{array}{l}\text { Anatomical, } \\
\text { physiological }\end{array}$ & $\begin{array}{l}\text { Physiological, } \\
\text { molecular }\end{array}$ \\
\hline Quantitative & Yes & Yes & No & Yes & Yes \\
\hline
\end{tabular}


Gd-DTPA-di(stearylamide). The presence of this high payload of Gd(III)-containing lipids ensures a high particulate relaxivity. In addition, the paramagnetic liposome was also encoded with $0.1 \mathrm{~mol} \%$ rhodamine fluorescent lipids. The final size of the liposome was $150 \mathrm{~nm}$. After being conjugated with cyclic arginine-glycine-aspartic acid (RGD) peptides, the liposomal contrast agent (RGD-liposomes) was used to identify the angiogenic endothelium in tumor-bearing mice. MRI indicated that the RGD liposomes localized to a large extent in the tumor rim after being injected intravenously. Nonspecific liposomes, liposomes coupled with scramble RAD peptides (RAD-liposomes), also targeted the tumor, but showed a diffuse distribution pattern. The different mechanisms of accumulation were established with fluorescence microscopy, which revealed that RGD-liposomes were exclusively associated with tumor blood vessels, whereas RAD-liposomes were mainly localized in the extravascular compartment. ${ }^{42}$

The above integrin $\alpha v \beta 3$-specific RGD-liposomes were also applied for noninvasive evaluation of the angiostatic therapy efficacy of anginex or endostatin for tumor by MRI. ${ }^{43}$ The percentage of enhanced pixels in the tumor on T1-weighted MRI after injection with RGD-liposomes served as a noninvasive in vivo readout of angiogenic activity. It was found to closely reflect the treatment effects as deduced from the ex vivo analyses. High density lipoprotein (HDL), an endogenous nanoparticle, is compatible with the immune system and can escape removal by the reticuloendothelial system. HDL nanoparticles have been used as carriers for the delivery of drugs to tumors, and have also been rerouted for tumor imaging. ${ }^{44}$ Chen et $\mathrm{a}^{45}$ have reconstituted HDL (rHDL) with amphiphilic Gd-DTPA and a fluorescent dye. After modification with RGD peptides (rHDL-RGD), rHDL-RGD was applied for targeting integrin $\alpha v \beta 3$ for depicting tumor angiogenesis. The hydrodynamic size of rHDL-RGD was $12.1 \pm 2.1 \mathrm{~nm}$, and the T1 relaxivity was $8.7 \pm 0.2 \mathrm{mM}^{-1} \mathrm{~s}^{-1}$, much higher than that of Gd-DTPA (Magnevist, $\sim 3 \mathrm{mM}^{-1} \mathrm{~s}^{-1}$ ). The specificity of rHDL-RGD for integrin $\alpha v \beta 3$ was demonstrated by incubating proliferating human umbilical vein endothelial cells (HUVECs, integrin $\alpha v \beta 3$-positive) with rHDL-RGD or control (scramble peptide RAD-encoded rHDL [rHDL-RAD] and plain rHDL). Both fluorescence imaging and MRI indicated that cell uptake of rHDL-RGD was more pronounced than those of rHDL-RAD and rHDL in the short term (less than 2 hours). Moreover, the uptake could be inhibited with competition with free RGD peptides. Introduced into mice bearing human EW7 Ewing's sarcoma intravenously, rHDL-RGD rapidly accumulated in tumors.
Confocal microscopy revealed that rHDL-RGD was associated with tumor endothelial cells, while rHDL and rHDLRAD were mainly found in the interstitial space. This study demonstrates that HDL is a potent platform for preparing high sensitivity molecular imaging probes for imaging of tumorassociated processes. Besides the abovementioned paramagnetic formulations, various other Gd(III)-containing small or macromolecules have been developed for T1-weighted imaging of tumor angiogenesis. ${ }^{46}$

\section{Imaging with single superparamagnetic iron oxide nanoparticles (SPIONs)}

SPIONs are the most widely used T2 (or T2*) contrast agent in MRI. ${ }^{47}$ A number of SPION-based MRI contrast agents have already been used in the clinical setting. ${ }^{48}$ We first reported MRI of tumor angiogenesis by RGD peptide-encoded SPIONs (RGD-SPIONs) with a clinical $1.5 \mathrm{~T}$ MRI scanner. ${ }^{49}$ The SPIONs were prepared by coprecipitation of $\mathrm{Fe}^{+2}$ and $\mathrm{Fe}^{+3}$ in the basic condition, modified with organic silane ((3-aminopropyl)trimethoxysilane) and $\mathrm{c}(\mathrm{RGDyE})$. The mean size of the particles as determined by transmission electron microscopy was $10 \pm 3 \mathrm{~nm}$. The surface charge of the particles was positive with an isoelectric point at $\mathrm{pH}$ 9.6. T2 relaxivity was $134 \mathrm{~s}^{-1}$ - $\mathrm{mM}^{-1}$. Compared to plain SPIONs, HUVECs had a higher uptake of RGD-SPIONs for both internalization through the cell membrane and accumulation within endosomes. Moreover, the uptake was reduced after inhibiting the cells with free RGD peptides, which verified the specificity of RGDSPIONs for integrin $\alpha v \beta 3$. In line with the in vitro observations, after intravenous injection of RGD-SPIONs into mice bearing squamous carcinoma (HaCaT-ras-A-5RT3 or A341), T2*-weighted MR imaging identified the heterogeneous distribution of $\alpha v \beta 3$-positive tumor vessels by an irregular signal intensity decrease in HaCaT-ras-A-5RT3 xenografts, whereas the signal intensity decreased more homogeneously in the control tumors (A431) with predominantly small and uniformly distributed vessels (Figure 1). This study indicated that integrin $\alpha v \beta 3$-targeted SPIONs were able to noninvasively differentiate tumors with high and lower area fractions of $\alpha v \beta 3$-positive tumor vessels. In an extended study, the same RGD-SPIONs were applied for the detection of tumor angiogenesis with different $\alpha v \beta 3$ expression profiles by a $3 \mathrm{~T}$ MRI scanner. ${ }^{50} \mathrm{R} 2 *$ pseudo-color images revealed that A549 tumor, a lung tumor with $\alpha v \beta 3$ expression on both tumor cells and tumor angiogenic vessels, had homogenous signal intensity decrease throughout the whole tumor area. Whereas, for 3LL tumor (a lung tumor with $\alpha v \beta 3$ expression only on tumor angiogenic vessels), MR signal intensity decrease was 
A

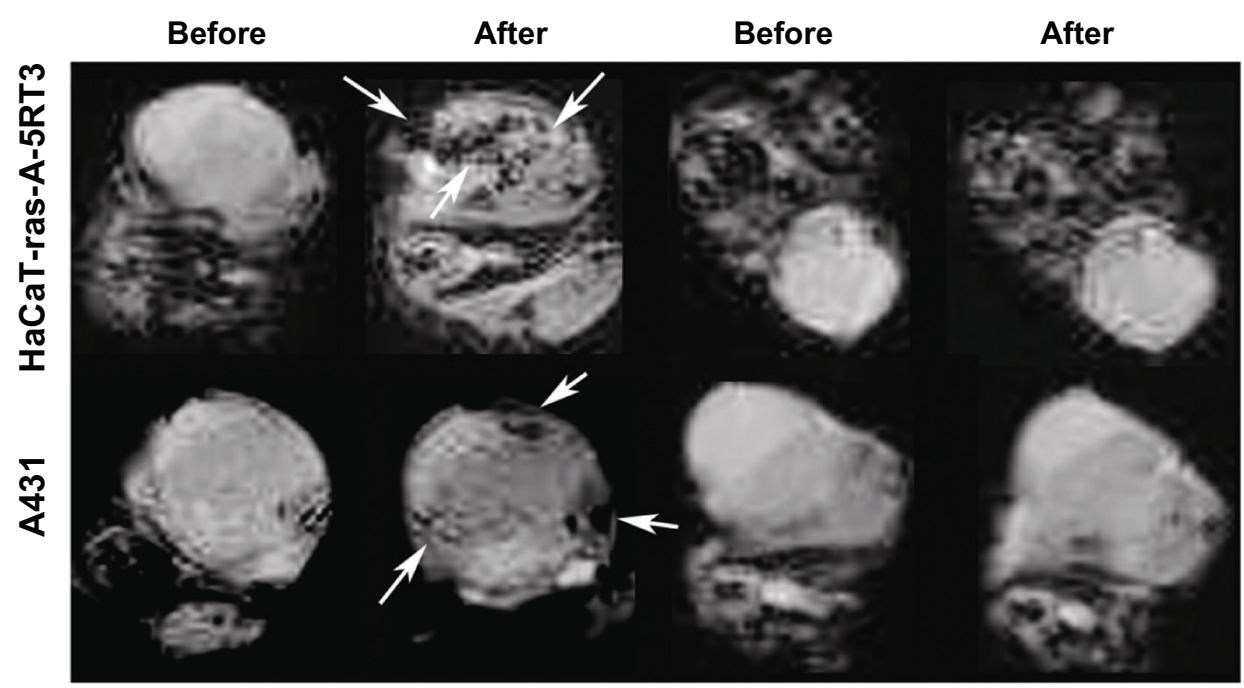

B

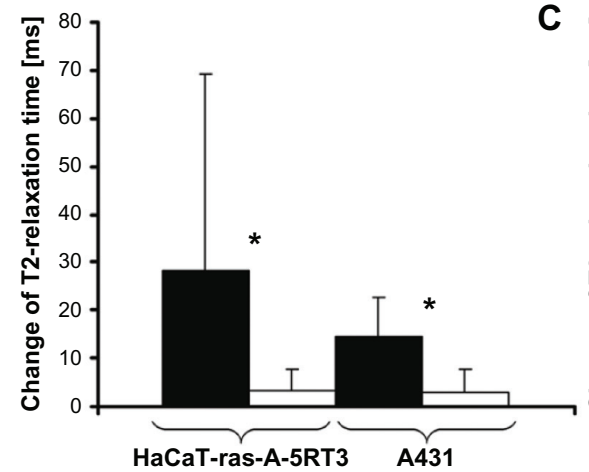

C

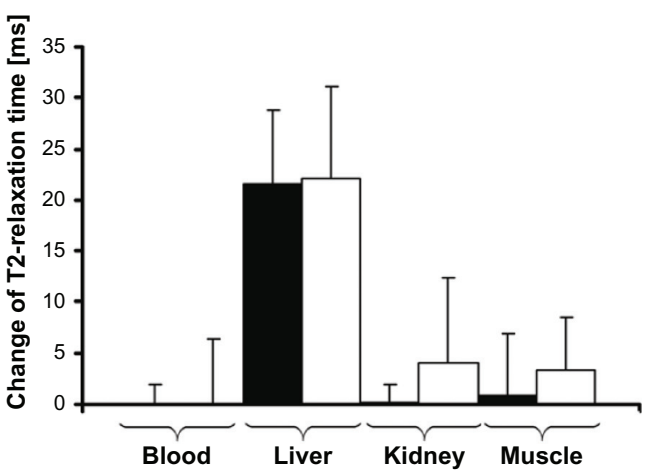

Figure I MR imaging of tumors with different $\alpha v \beta 3$ expression profiles. (A) T2*-weighted MR images of nude mice bearing subcutaneous HaCaT-ras-A-5RT3 (top row) and A43I tumors (bottom row) before and 6 hours after iv injection of RGD-SPIONs and SPIONs, respectively. In HaCaT-ras-A-5RT3 tumors, focal areas with strong and heterogeneous signal intensity decrease are observed in the tumor center and in the periphery after injection of RGD-SPIONs (arrows). Only a few spots with high susceptibility are found at the margin of the control tumor after injection of SPIONs. Signal intensity changes in the A43I tumor are much less pronounced and more homogeneous, and focal areas with a strong signal intensity decrease are only found at the tumor margins (arrows). No signal changes are observed in control tumors by visual inspection. (B) Change of T2 relaxation times in HaCaT-ras-A-5RT3 $(n=8)$ and A43I $(n=6)$ tumors after injection of RGD-SPIONs and SPIONs, respectively. Absolute T2 relaxation times before/after injection with RGD-SPIONs were $124.44 \pm 55.09 / 94.09 \pm 17.12 \mathrm{~ms}$ in HaCaT-ras-A-5RT3 and I05.66 \pm 10.67/90.16 \pm 7.84 ms in A43I tumors. The values for injection with SPIONs were $95.97 \pm 16.97 / 93.74 \pm 15.86 \mathrm{~ms}$ (HaCaT-ras-A-5RT3) and 106.47 \pm 13.16/101.46 $\pm 13.28 \mathrm{~ms}$ (A43I tumors), respectively. *P $<0.05$. (C) Change of T2 relaxation times in blood, liver, kidney, and muscle of A43I tumor-bearing mice after injection of RGD-SPIONs $(n=6)$ and SPIONs $(n=6)$, respectively.Absolute T2 relaxation times before/after injection of RGDSPIONs were $101.8 \pm 17.58 / 101.76 \pm 17.77 \mathrm{~ms}$ in blood, $53.06 \pm 3.8 \mathrm{I} / 31.56 \pm 5.54 \mathrm{~ms}$ in liver, $90.53 \pm 10.05 / 90.4 \pm 8.60 \mathrm{~ms}$ in kidney, and $51.33 \pm 8.88 / 50.40 \pm 5.1 \mathrm{I} \mathrm{ms}$ in muscle. Notes: For SPIONs, the values were $77.26 \pm 15.73 / 79.28 \pm 10.90 \mathrm{~ms}$ in blood, $56.66 \pm 7.90 / 37.33 \pm 4.43 \mathrm{~ms}$ in liver, $82.26 \pm 17.37 / 70.90 \pm 17.36 \mathrm{~ms}$ in kidney, and $50.40 \pm 5.68 / 46.04 \pm 3.78 \mathrm{~ms}$ in muscle.

(C) 2007. American Assocation for Cancer Research. Reproduced with permission from Zhang C, Jugold M, Woenne EC, et al. Specific targeting of tumor angiogenesis by RGD-conjugated ultrasmall superparamagnetic iron oxide particles using a clinical I.5-T magnetic resonance scanner. Cancer Res. 2007;67(4):I555-I562.49

Abbreviations: RGD, arginine-glycine-aspartic acid; SPION, superparamagnetic iron oxide nanoparticles; MR, magnetic resonance; iv, intravenous.

only distributed on the tumor periphery. The authors concluded that in addition to targeting tumor angiogenic vessels, RGDSPIONs could also extravasate from the vessels, addressing $\alpha v \beta 3$-positive tumor cells.

For the preparation of water-soluble SPIONs, the coprecipation method often leads to SPIONs with poorly defined nanocrystalline size, stoichiochemistry, and magnetism, which in turn influence the quality of MR signals. ${ }^{51}$ Alternatively, high-quality, near-monodisperse SPIONs can be prepared by thermal decomposition of iron organic precursors, such as $\mathrm{Fe}(\mathrm{acac})_{3}, \mathrm{Fe}(\mathrm{CO})_{3}$, and $\mathrm{Fe}(\text { oleic acid })_{3}$, at high temperatures in the presence of surfactants (oleic acid and oleylamine). ${ }^{52,53}$ However, these SPIONs cannot be biofunctionalized as they are coated with hydrophobic surfactants and are water-insoluble, and do not have suitable functional groups for bioconjugation. In this context, $\mathrm{Xu}$ et $\mathrm{al}^{54}$ have developed a general strategy to fabricate an MR molecular imaging probe in one step by replacing hydrophobic surfactants with small peptides terminated with cysteine, such as RGD-Cys and RGD-PEGCys (Figure 2). The hydrodynamic sizes of RGD-Cys- and RGD-PEG-Cys-SPIONs were $11 \mathrm{~nm}$ and $13 \mathrm{~nm}$, respectively. The in vitro studies indicated that RGD-Cys-SPIONs and 


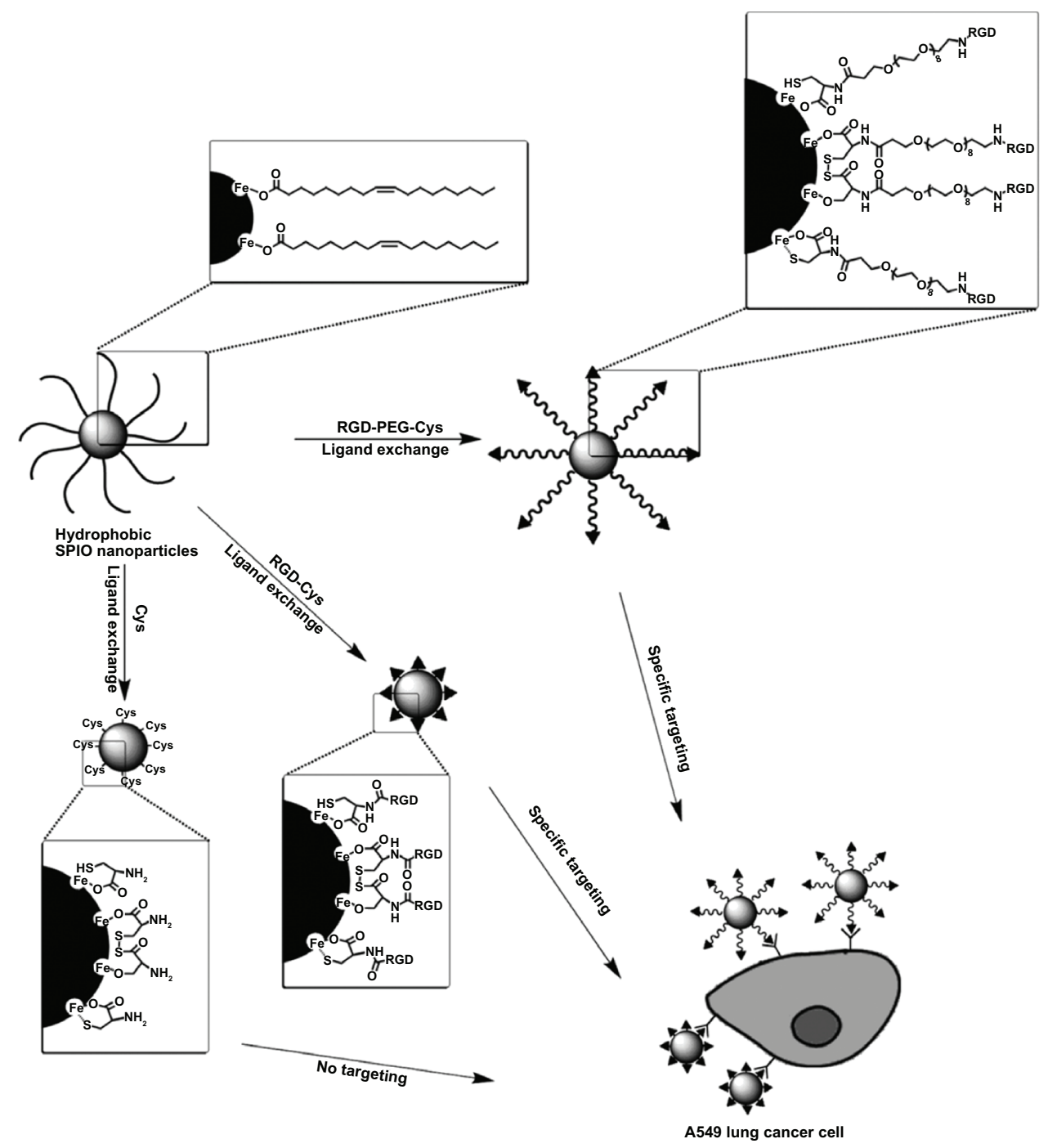

Figure 2 Scheme for one-step ligand exchange method for the preparation of an $\alpha v \beta 3$-targeted probe. Reproduced with permission. ${ }^{55}$

RGD-PEG-Cys-SPIONs specifically targeted $\alpha v \beta 3$-positive cells (A549), with RGD-Cys-SPIONs achieving this more efficiently. Administered into mice bearing A549 nonsmall cell lung tumor intravenously, both RGD functionalized SPIONs were able to reach and label the $\alpha v \beta 3$-expressing tumors, much more efficiently than nonpeptide functionalized particles (Cys-SPIONs). Histological studies of tumor tissues showed that the probes not only targeted the tumor neovasculature, but also extravasated from vessels, homing to the tumor cells. The efficacy of the ligand exchange method for the preparation of small peptide-modified SPION probes was also corroborated by another study. ${ }^{55}$

\section{Imaging with SPION clusters}

Sensitive molecular imaging and detection of tumors or their supporting neovascularity require high avidity, target-specific probes that produce robust signal amplification. Clustering individual SPIONs into SPION clusters can dramatically enhance the MR sensitivity of SPION contrast agents, ${ }^{56-58}$ which makes it possible for the detection of tumor angiogenesis at a very early stage. ${ }^{59}$ In this context, Nasongkla et al ${ }^{60}$ have developed multifunctional SPION-containing polymeric micelles by the assembly of hydrophobic SPIONs with amphiphilic copolymers, and further modified the micelles with RGD peptides (c(RGDfK)) for the detection of integrin $\alpha v \beta 3$. The mean size of the micelles was $46 \mathrm{~nm}$. Clustering SPIONs inside micelle cores has demonstrated the dramatic increase in T2 relaxivity (per Fe). As a result, in a phantom study, picogram concentrations of the micelles were detected. ${ }^{61}$ In a following study, the same group fabricated SPION micelles with a high SPION payload (33 wt \%) using amphiphilic poly(ethylene glycol)-block-poly(D,L-lactide) 
copolymer (PEG-PLA; molecular weights for PEG and PLA blocks are $5 \mathrm{kDa})$ and hydrophobic SPIONs $(9.9 \pm 0.4 \mathrm{~nm}$ in diameter). The sizes of the micelles were $75 \pm 11 \mathrm{~nm}$ determined by transmission electron microscope. After encoding with cyclic (RGDfK) peptides denoted as cRGD-encoded superparamagnetic polymeric micelles (SPPM), the micelles were administered into mice with A549 nonsmall cell lung tumors. The neovasculature of the tumors was clearly manifested by a $4.7 \mathrm{~T}$ animal MRI scanner, and the imaging efficacy was further enhanced by an off-resonance saturation technique. ${ }^{62}$ Tumor accumulation of cRGD-encoded SPPM was corroborated by a biodistribution study, which indicated that tumor uptake of cRGD-encoded SPPM $(1.3 \% \pm 0.3 \%$ $\mathrm{ID} / \mathrm{g}$ ) was significantly higher than that of $\mathrm{cRGD}$-free SPPM $(0.6 \% \pm 0.3 \% \mathrm{ID} / \mathrm{g})$. However, most of the micelles were sequestered by spleen $(\sim 10 \% \mathrm{ID} / \mathrm{g})$ and liver $(\sim 4 \%$ $\mathrm{ID} / \mathrm{g})$. Plasma clearance of the micelles showed a two-phase behavior over 24 hours. The plasma half-lives for the initial phase were $0.34 \pm 0.09$ hours for cRGD-encoded SPPM and $0.40 \pm 0.34$ hours for cRGD-free SPPM. Those for the slow clearance phase were $3.9 \pm 0.8$ hours and $9.2 \pm 0.8$ hours for the cRGD-encoded and cRGD-free SPPM, respectively.

Recently, our group has fabricated high MR-sensitive SPION cluster probes specific for tumor angiogenesis with a similar approach: assembling hydrophobic SPIONs with amphiphilic PEG-PLA copolymers into clusters and subsequently conjugating $\mathrm{c}(\mathrm{RGDyC})$ peptide on the clusters (RGDmagnetite nanoclusters (MNC)) for the detection of nascent tumors. ${ }^{59}$ As-synthesized RGD-MNCs were fairly monodispersed with size about $62 \mathrm{~nm}$, highly sensitive $\left(\mathrm{r}_{2}=464.94 \mathrm{~s}^{-1}\right.$ $\mathrm{mM}^{-1}$ ), and specific for $\alpha v \beta 3$-positive cells. After being intravenously introduced into mice bearing H1299 lung tumors at different growth stages, both nascent $\left(35 \pm 6.6 \mathrm{~mm}^{3}\right)$ and big tumors $\left(256 \pm 22.3 \mathrm{~mm}^{3}\right)$ were registered by RGDMNCs and detected by a clinical MRI (3T) scanner, with the nascent tumors demonstrating more pronounced MR contrast (Figure 3). Consistent with MRI findings, biodistribution studies indicated that the accumulation of RGD-MNCs by nascent tumors was more significant than that by large ones $(5.83 \% \pm 1.01 \% \mathrm{ID} / \mathrm{g}$ versus $3.42 \% \pm 0.93 \% \mathrm{ID} / \mathrm{g}$, $P<0.05)$. However, the uptake by the liver $(32.05 \% \pm 2.31 \%$ $\mathrm{ID} / \mathrm{g}$ for nascent tumors, $35.21 \% \pm 6.10 \% \mathrm{ID} / \mathrm{g}$ for large tumors ) and lung $(24.66 \% \pm 3.78 \% \mathrm{ID} / \mathrm{g}$ for nascent tumors, $23.01 \% \pm 4.51 \% \mathrm{ID} / \mathrm{g}$ for large tumors) were dominant for both tumor groups. Immunohistochemical studies revealed that the MR signal decrease was closely correlated with the histological characteristics of the tumors (ie, microvessel densities and $\alpha v \beta 3$ expression levels) at different growth stages. This study demonstrated that MR molecular imaging with highly sensitive RGD-MNC probes presented a significant opportunity for the early detection of tumors and the possible characterization of tumor angiogenesis at different tumor growth stages.

\section{Imaging with dual-targeting probes}

In order to further improve the specificity of MR molecular imaging probes for tumor angiogenesis, a dual-targeting approach has been proposed. ${ }^{63}$ Cyclic RGD peptide and anginex (Anx), targeting integrin $\alpha v \beta 3$ and galectin-1, respectively, were simultaneously conjugated to paramagnetic liposomes (Anx/RGD-L). The size of the dual targeting liposomes was about $200 \mathrm{~nm}$. Compared with single peptide coupled liposomes, Anx-L or RGD-L, the specificity of Anx/RGD-L for activated endothelial cells was significantly improved both in vitro and in vivo due to the synergistic targeting effects of the two kinds of specific ligands. ${ }^{64}$ However, because of its relatively short circulation time (half-life, less than 0.5 hours for RGD/Anx-L and 11.2 \pm 2.5 hours for RGD-L), the targeting efficiency of RGD/Anx-L was $16 \%$ less than that of RGD-L 24 hours postinjection. Inductively coupled plasma mass spectrometry measurements of the organ-associated Gd(III) 24 hours postinjection revealed significant differences in the contrast agent biodistribution. Anx/RGD-L or Anx-L had similar tumor Gd(III) levels of $2.5 \pm 0.7 \mu \mathrm{g} / \mathrm{g}$ tissue, significantly lower than those found in the tumors of mice that received RGD-L $(18 \pm 11 \mu \mathrm{g} / \mathrm{g})$. However, the accumulation of both types of Anx-containing liposomes in the spleen was approximately $40 \%$ higher compared to RGD-L, whereas the liver uptake was similar in all cases.

\section{Biological effects of RGD peptide- conjugated probes on tumor cells}

The biological effects of integrin-specific probes on $\alpha v \beta 3$ positive tumor cells or endothelial cells are less studied. Recently, Kiessling et $a 1^{65}$ have prepared 3-aminopropyltrimethoxysilane-coated SPIONs. The particles were $10 \pm 3 \mathrm{~nm}$ and positively charged. After functionalized with RGD peptide (RGD-SPIONs), they found that RGD-SPIONs had differential effects on HUVECs, ovarian carcinoma (MLS) cells, and glioblastoma (U87MG) cells. RGD-SPIONs induced U87MG cells turning round and losing contacts with each other, which led to cell uptake of RGD-SPIONs less than that of plain SPIONs. However, RGD-SPIONs had no such effects on HUVECs and ovarian carcinoma cells, and both kinds of cells internalized more RGD-SPIONs than 

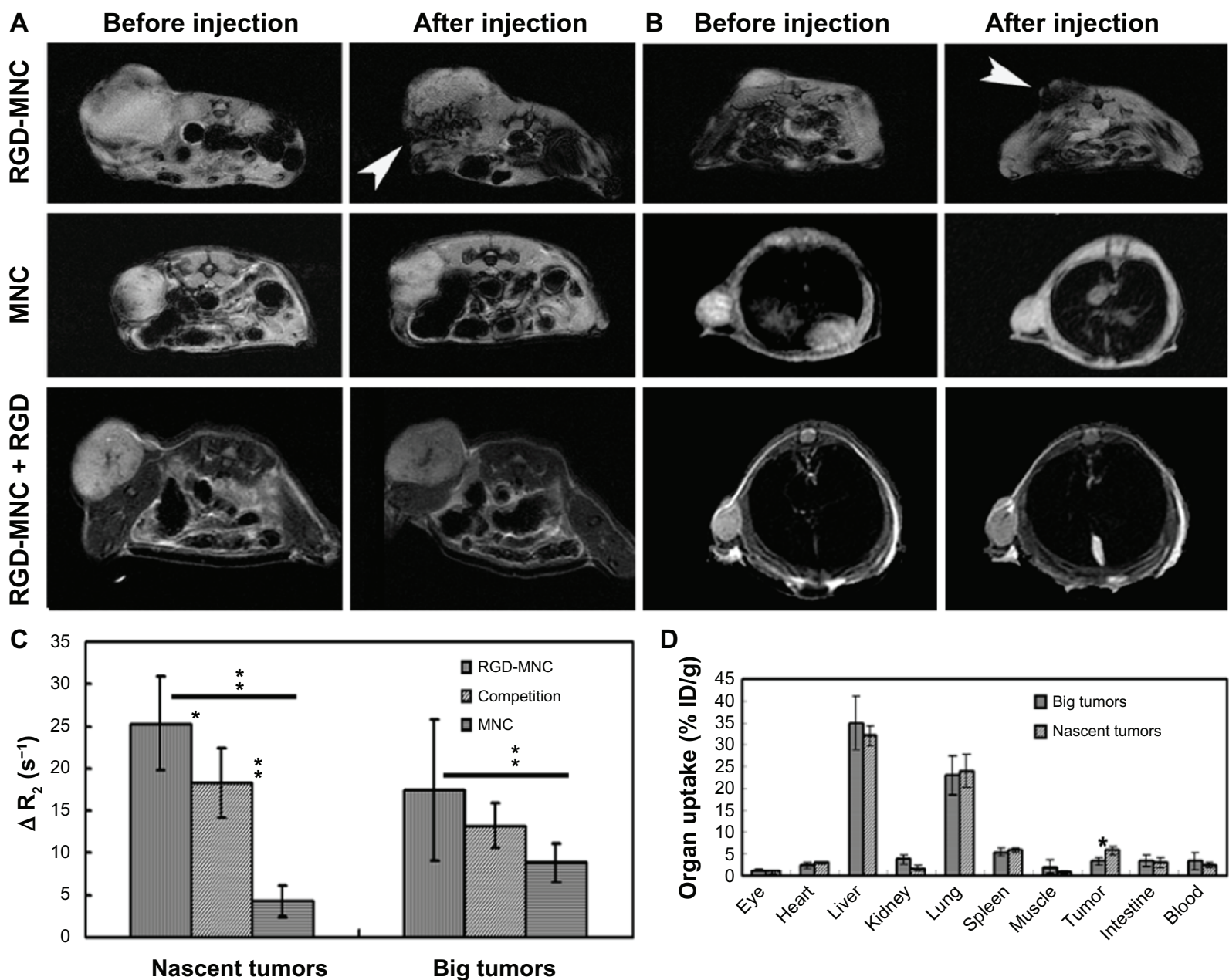

Figure 3 MR detection of tumors with RGD-MNC and its biodistribution. $T_{2}$-weighted MR images of (A) big tumors and (B) nascent tumors before and after iv injection of RGD-MNC, MNC, or RGD-MNC plus free RGD peptide at the dose of $200 \mu \mathrm{mol} \mathrm{Fe} / \mathrm{kg}$. For competition study, free RGD peptide (I00 $\mu \mathrm{L}$, $0.15 \mathrm{mM}$ ) was injected I hour before RGD-MNC administration. (C) The corresponding $T_{2}$ relaxation rate changes of tumors after and before probe injection. $* P<0.05$, $* * P<0.01$. (D) Biodistribution of iodine- 125 labeled RGD-MNC in nascent and big tumor groups 12 hours postinjection.

(C20 I2. Elsevier. Reproduced with permission from Zhang C, Xie X, Liang S, Li M, Liu Y, Gu H. Mono-dispersed high magnetic resonance sensitive magnetite nanocluster probe for detection of nascent tumor by magnetic resonance molecular imaging. Nanomedicine: Nanotechnology, Biology, and Medicine. 2012;8(6):996-1006. ${ }^{59}$

Abbreviations: MR, magnetic resonance; RGD, arginine-glycine-aspartic acid; MNC, magnetite nanoclusters; iv, intravenous.

plain SPIONs (Figure 4). T2*-weighted MR imaging indicated that tumors accumulated RGD-SPIONs significantly more than plain SPIONs. However, histological studies of tumor tissues identified RGD-SPIONs mainly constrained on the tumor angiogenic vessels, not addressing tumor cells (U87MG). The authors ascribed this to the differential effects of RGD-SPIONs on tumor cells and endothelial cells. Kluza et al ${ }^{63}$ have observed that liposomes functionalized with anti- $\alpha v \beta 3$ integrin RGD peptide (RGD-L), antigalectin-1 peptide (Anx-L), or both of them simultaneously (Anx/RGD-L) affected the proliferation of HUVECs and decreased the cell number both in the $\mathrm{S}$ phase and in the G2/M phase, with Anx/RGD-L more significantly. Based on these findings, they suggested that Anx/RGD-L could be used for therapeutic purposes. These studies demonstrate that if molecular imaging is performed for the detection of tumor angiogenesis with $\alpha v \beta 3$ integrin-targeted probes, biologic effects of the probes must be considered, which can be highly divergent between different cells and may lead to different targeting sites of the probes.

\section{Summary and future perspectives}

In the current review, we summarized the advance in MR molecular imaging of tumor angiogenesis by targeting integrin $\alpha v \beta 3$ with RGD peptide modified magnetic probes. For detection of the sparse angiogenic marker, the imaging probes evolved from paramagnetic nanoparticles to high sensitive SPIONs, and further to ultrahigh sensitive SPION clusters. With SPION cluster probes, nascent tumors could be detected and MR signal changes were closely correlated 

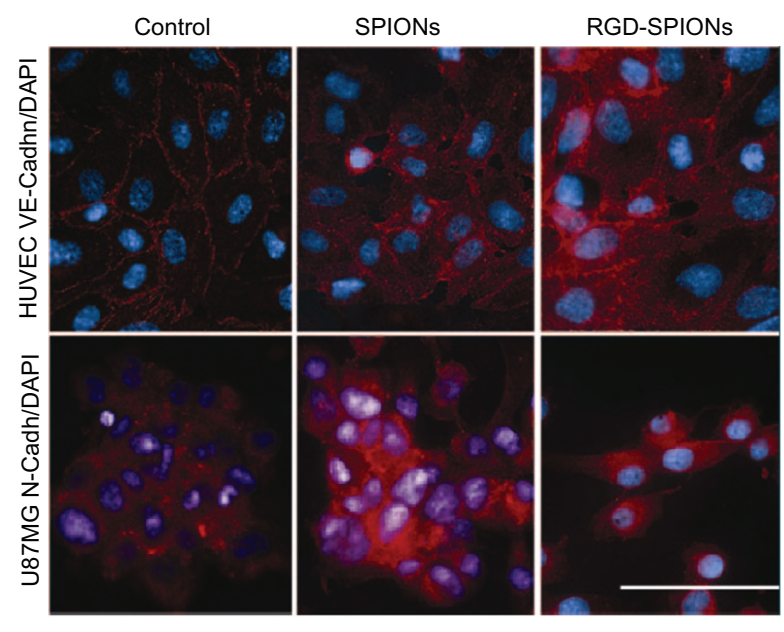

Figure 4 Immunofluorescence microscopy of HUVECs and U87MG cells. Notes: Staining (red) was performed against VE-cadh (HUVEC) and N-cadh (U87MG) cells. Cell nuclei were stained with DAPI (blue). In HUVECs incubated with regular growth medium containing SPIONs or not, VE-cadh was located along the lateral cell membrane, which results in a netlike pattern of the cell monolayer at fluorescence microscopy. After RGD-SPIONs incubation, this pattern is still present; however, an enhanced perinuclear staining also occurs. In U87MG cells incubated with regular growth medium in presence of SPIONs or not, intercellular contacts are more punctual. While $\mathrm{N}$-cadh is most strongly expressed at intercellular contact zones, it can be located in other parts of the plasma membrane and perinuclearly. After incubation with RGD-SPIONs, however, cells lose most of their intercellular contacts, round up, and separate from each other. Bar $=20 \mu \mathrm{m}$.

(C) 2009. The Radiological Society of North America (RSNA ${ }^{\circledR}$ ). Reproduced with permission from Kiessling F, Huppert J, Zhang C, et al. RGD-labeled USPIO inhibits adhesion and endocytotic activity of alpha $v$ beta3-integrin-expressing glioma cells and only accumulates in the vascular tumor compartment. Radiology. 2009;253(2):462-469.65 Abbreviations: HUVECs, human umbilical vein endothelial cells; SPIONs, superparamagnetic iron oxide nanoparticles; RGD, arginine-glycine-aspartic acid; DAPI, 4,6-diamidino-2-phenylindole; VE-Cadh, VE-cadherin; N-Cadh, N-cadherin.

with histological characteristics of tumors (ie, microvessel densities and $\alpha v \beta 3$ expression levels) at different growth stages. In order to further improve the detection specificity, dual-targeting approach, targeting two different angiogenic markers simultaneously, was also proposed. However, the pharmacodynamics of the probes must be carefully evaluated before application. In addition, more attention should be paid on the biological effects of angiogenesis targeting probes on tumor cells, which may affect their ultimate targeting sites. Taking these results together, MR molecular imaging of angiogenesis for the early detection of tumors is very promising; however, there is still need for improvement of pharmacokinetics, specific binding, and signaling of the probes.

One disadvantage of MRI is its inherently low sensitivity to contrast agents. ${ }^{66}$ Most efforts for improving the detection sensitivity are to enhance the sensitivity and specificity of imaging probes and, as summarized above, tremendous progress has been achieved in this direction. However, currently radionuclide-based imaging techniques (eg, SPECT and PET) are still the most sensitive and highly quantitative methods. ${ }^{67}$
Nevertheless, MRI can provide high-resolution anatomic information. Therefore, dual-modality imaging probes combining both MRI and radionuclide-based imaging properties in one unit should be of particular interest for tumor angiogenesis imaging. Especially with the development of a PET/MRI integrated system and its progression to clinical application, ${ }^{68-70}$ the simultaneous acquisition of PET and MRI data is possible, which would create enormous possibilities and provide completely new opportunities to study pathology and biochemical processes in vivo. Therefore, PET/MR contrast agents deserve much more research effort in this field in the near future.

\section{Acknowledgments}

This work was supported by the Major State Basic Research Development Program of China (973 Program; number 2010CB834303), the National Nature Science Foundation of China (81230030, 30870682), the Med-Engineering Crossing Foundation from Shanghai Jiao Tong University (YG2011ZD07, YG2012MS15), SRF for ROCS, and the Research Fund for the Doctoral Program of Higher Education of China (20090073120004).

\section{Disclosure}

The authors report no conflicts of interest in this work.

\section{References}

1. Carmeliet P. Mechanisms of angiogenesis and arteriogenesis. Nat Med. 2000;6(4):389-395.

2. Folkman J. Anti-angiogenesis: new concept for therapy of solid tumours. Ann Surg. 1972;175(3):409-416.

3. Weidner N, Semple JP, Welch WR, Folkman J. Tumor angiogenesis and metastasis - correlation in invasive breast carcinoma. $N$ Engl $J$ Med. 1991;324(1):1-8.

4. Chiang AC, Massaqué J. Molecular basis of metastasis. N Engl J Med. 2008;359(26):2814-2823.

5. Folkman J. Tumor angiogenesis: therapeutic implications. $N$ Engl J Med. 1971;285(21):1182-1186.

6. Hagedorn M, Bikfalvi A. Target molecules for anti-angiogenic therapy: from basic research to clinical trials. Crit Rev Oncol Hematol. 2000;34(2):89-110.

7. Folkman J, Klagsbrun M. Angiogenic factors. Science. 2001; 235(4787):442-447.

8. Rundhaug JE. Matrix metalloproteinases and angiogenesis. J Cell Mol Med. 2005;9(2):267-285.

9. Yancopoulos GD, Davis S, Gale NW, Rudge JS, Wiegand SJ, Holash J. Vascular-specific growth factors and blood vessel formation. Nature. 2000;407(6801):242-248.

10. Nussenbaum F, Herman IM. Tumor angiogenesis: insights and innovations. J Oncol. 2010;2010:132641.

11. Auguste P, Lemiere S, Larrieu-Lahargue F, Bikfalvi A. Molecular mechanisms of tumor vascularization. Crit Rev Oncol Hematol. 2005;54(1):53-61.

12. Ramjaun AR, Hodivala-Dilke K. The role of cell adhesion pathways in angiogenesis. Int J Biochem Cell Biol. 2009;41(3):521-530.

13. Francavilla $C$, Maddaluno L, Cavallaro U. The functional role of cell adhesion molecules in tumor angiogenesis. Semin Cancer Biol. 2009;19(5):298-309. 
14. Snoeks TJ, Löwik CW, Kaijzel EL. 'In vivo' optical approaches to angiogenesis imaging. Angiogenesis. 2010;13(2):135-147.

15. Mahmoudi M, Serpooshan V, Laurent S. Engineered nanoparticles for biomolecular imaging. Nanoscale. 2011;3(8):3007-3026.

16. Ruoslahti E. RGD and other recognition sequences for integrins. Annu Rev Cell Dev Biol. 1996;12:697-715.

17. Cai W, Sam Gambhir S, Chen X. Multimodality tumor imaging targeting integrin alphavbeta3. Biotechniques. 2005;39(Suppl 6): S14-S25.

18. Liu S. Radiolabeled cyclic RGD peptides as integrin alpha(v)beta(3) targeted radiotracers: maximizing binding affinity via bivalency. Bioconjug Chem. 2009;20(12):2199-2213.

19. Danhier F, Le Breton A, Préat V. RGD-based strategies to target alpha(v) beta(3) integrin in cancer therapy and diagnosis. Mol Pharm. 2012;9(11):2961-2973.

20. Hood JD, Cheresh DA. Role of integrins in cell invasion and migration. Nat Rev Cancer. 2002;2(2):91-100.

21. Brooks PC, Clark RA, Cheresh DA. Requirement of vascular integrin alpha v beta 3 for angiogenesis. Science. 1994;264(5158):569-571.

22. Gladson CL. Expression of integrin alpha $v$ beta 3 in small blood vessels of glioblastoma tumors. J Neuropathol Exp Neurol. 1996; 55(11):1143-1149.

23. Liu Z, Peng R. Inorganic nanomaterials for tumor angiogenesis imaging. Eur J Nucl Med Mol Imaging. 2010;37 Suppl 1:S147-S163.

24. Zhang Y, Yang Y, Cai W. Multimodality imaging of integrin $\alpha(\mathrm{v}) \beta(3)$ expression. Theranostics. 2011;1:135-148.

25. Beer AJ, Schwaiger M. Imaging of integrin alphavbeta3 expression. Cancer Metastasis Rev. 2008;27(4):631-644.

26. Contag $\mathrm{CH}$. In vivo pathology: seeing with molecular specificity and cellular resolution in the living body. Annu Rev Pathol. 2007;2: 277-305.

27. Kim HL. Optical imaging in oncology. Urol Oncol. 2009;27(3): 298-300.

28. Deshpande N, Pysz MA, Willmann JK. Molecular ultrasound assessment of tumor angiogenesis. Angiogenesis. 2010;13(2):175-188.

29. Kobayashi H, Longmire MR, Ogawa M, Choyke PL. Rational chemical design of the next generation of molecular imaging probes based on physics and biology: mixing modalities, colors and signals. Chem Soc Rev. 2011;40(9):4626-4648.

30. Kwee TC, Takahara T, Ochiai R, et al. Whole-body diffusion-weighted magnetic resonance imaging. Eur J Radiol. 2009;70(3):409-417.

31. Takenaka D, Ohno Y, Matsumoto K, et al. Detection of bone metastases in non-small cell lung cancer patients: comparison of whole-body diffusion-weighted imaging (DWI), whole-body MR imaging without and with DWI, whole-body FDG-PET/CT, and bone scintigraphy. J Magn Reson Imaging. 2009;30(2):298-308.

32. Tsushima Y, Takano A, Taketomi-Takahashi A, Endo K. Body diffusion-weighted MR imaging using high $b$-value for malignant tumor screening: usefulness and necessity of referring to T2-weighted images and creating fusion images. Acad Radiol. 2007;14(6):643-650.

33. He Q, Xu RZ, Shkarin P, et al. Magnetic resonance spectroscopic imaging of tumor metabolic markers for cancer diagnosis, metabolic phenotyping, and characterization of tumor microenvironment. Dis Markers. 2004;19(2-3):69-94.

34. Caravan P, Ellison JJ, McMurry TJ, Lauffer RB. Gadolinium(III) chelates as MRI contrast agents: structure, dynamics, and applications. Chem Rev. 1999;99(9):2293-2352.

35. Sipkins DA, Cheresh DA, Kazemi MR, Nevin LM, Bednarski MD, Li KC. Detection of tumor angiogenesis in vivo by alphaVbeta3-targeted magnetic resonance imaging. Nat Med. 1998;4(5):623-626.

36. Lanza GM, Winter PM, Caruthers SD, et al. Nanomedicine opportunities for cardiovascular disease with perfluorocarbon nanoparticles. Nanomedicine (Lond). 2006;1(3):321-329.

37. Winter PM, Caruthers SD, Kassner A, et al. Molecular imaging of angiogenesis in nascent Vx-2 rabbit tumors using a novel alpha(nu) beta3-targeted nanoparticle and 1.5 tesla magnetic resonance imaging. Cancer Res. 2003;63(18):5838-5843.
38. Schmieder AH, Winter PM, Caruthers SD, et al. Molecular MR imaging of melanoma angiogenesis with alphanubeta3-targeted paramagnetic nanoparticles. Magn Reson Med. 2005;53(3):621-627.

39. Mulder WJ, Strijkers GJ, van Tilborg GA, Griffioen AW, Nicolay K. Lipid-based nanoparticles for contrast-enhanced MRI and molecular imaging. NMR Biomed. 2006;19(1):142-164.

40. Morawski AM, Winter PM, Crowder KC, et al. Targeted nanoparticles for quantitative imaging of sparse molecular epitopes with MRI. Magn Reson Med. 2004;51(3):480-486.

41. Strijkers GJ, Kluza E, Van Tilborg GA, et al. Paramagnetic and fluorescent liposomes for target-specific imaging and therapy of tumor angiogenesis. Angiogenesis. 2010;13(2):161-173.

42. Mulder WJ, Strijkers GJ, Habets JW, et al. MR molecular imaging and fluorescence microscopy for identification of activated tumor endothelium using a bimodal lipidic nanoparticle. FASEB J. 2005;19(14): 2008-2010

43. Mulder WJ, van der Schaft DW, Hautvast PA, et al. Early in vivo assessment of angiostatic therapy efficacy by molecular MRI. FASEB $J$. 2007;21(2):378-383.

44. Gianella A, Read JC, Cormode DP, Fayad ZA, Mulder WJM. Multifunctional nanoparticles for target-specific imaging and therapy. In: Svenson S, Prud'homme K, editors. Multifunctional Nanoparticles for Drug Delivery Applications. New York: Springer. 2012:155-171.

45. Chen W, Jarzyna PA, van Tilborg GA, et al. RGD peptide functionalized and reconstituted high-density lipoprotein nanoparticles as a versatile and multimodal tumor targeting molecular imaging probe. FASEB $J$. 2010;24(6):1689-1699.

46. Tan M, Lu ZR. Integrin targeted MR imaging. Theranostics. 2011;1: 83-101.

47. Tassa C, Shaw SY, Weissleder R. Dextran-coated iron oxide nanoparticles: a versatile platform for targeted molecular imaging, molecular diagnostics, and therapy. Acc Chem Res. 2011;44(10):842-852.

48. Weinstein JS, Varallyay CG, Dosa E, et al. Superparamagnetic iron oxide nanoparticles: diagnostic magnetic resonance imaging and potential therapeutic applications in neurooncology and central nervous system inflammatory pathologies, a review. J Cereb Blood Flow Metab. 2010;30(1):15-35.

49. Zhang C, Jugold M, Woenne EC, et al. Specific targeting of tumor angiogenesis by RGD-conjugated ultrasmall superparamagnetic iron oxide particles using a clinical 1.5-T magnetic resonance scanner. Cancer Res. 2007;67(4):1555-1562.

50. Jiang $\mathrm{T}$, Zhang $\mathrm{C}$, Zheng $\mathrm{X}$, et al. Noninvasively characterizing the different alphavbeta3 expression patterns in lung cancers with RGD-USPIO using a clinical 3.0T MR scanner. Int J Nanomedicine. 2009;4:241-249.

51. Sjögren CE, Johansson C, Naevestad A, Sontum PC, Briley-Saebø K, Fahlvik AK. Crystal size and properties of superparamagnetic iron oxide (SPIO) particles. Magn Reson Imaging. 1997;15(1):55-67.

52. Ho D, Sun X, Sun S. Monodisperse magnetic nanoparticles for theranostic applications. Acc Chem Res. 2011;44(10):875-882.

53. Jeong U, Teng X, Wang Y, Yang H, Xia Y. Superparamagnetic colloids: controlled synthesis and niche application. Advanced Materials. 2007; 19(1):33-60.

54. Xu F, Lei D, Du X, Zhang C, Xie X, Yin D. Modification of MR molecular imaging probes with cysteine-terminated peptides and their potential for in vivo tumour detection. Contrast Media Mol Imaging. 2011;6(1):46-54.

55. Huang G, Zhang C, Li S, et al. A novel strategy for surface modification of superparamagnetic iron oxide nanoparticles for lung cancer imaging. J Mater Chem. 2009;19:6367-6372.

56. Xie X, Zhang C. Controllable assembly of hydrophobic superparamagnetic iron oxide nanoparticle with mPEG-PLA copolymer and its effect on MR transverse relaxation rate. J Nanomater. 2010;2011:Article ID 152524 .

57. Xu F, Cheng C, Xu F, et al. Superparamagnetic magnetite nanocrystal clusters: a sensitive tool for MR cellular imaging. Nanotechnology. 2009;20(40):405102. 
58. Wang $\mathrm{Y}, \mathrm{Xu} \mathrm{F}$, Zhang $\mathrm{C}$, et al. High MR sensitive fluorescent magnetite nanocluster for stem cell tracking in ischemic mouse brain. Nanomedicine: Nanotechnology, Biology, and Medicine. 2011;7(6): 1009-1019.

59. Zhang C, Xie X, Liang S, Li M, Liu Y, Gu H. Mono-dispersed high magnetic resonance sensitive magnetite nanocluster probe for detection of nascent tumor by magnetic resonance molecular imaging. Nanomedicine: Nanotechnology, Biology, and Medicine . 2012;8(6): 996-1006.

60. Nasongkla N, Bey E, Ren J, et al. Multifunctional polymeric micelles as cancer-targeted, MRI-ultrasensitive drug delivery systems. Nano Lett. 2006;6(11):2427-2430.

61. Ai H, Flask C, Weinberg B, et al. Magnetite-loaded polymeric micelles as ultrasensitive magnetic-resonance probes. Advanced Materials. 2005;17(16):1949-1952.

62. Khemtong C, Kessinger CW, Ren J, et al. In vivo off-resonance saturation magnetic resonance imaging of alphavbeta3-targeted superparamagnetic nanoparticles. Cancer Res. 2009;69(4):1651-1658.

63. Kluza E, van der Schaft DW, Hautvast PA, et al. Synergistic targeting of alphavbeta3 integrin and galectin-1 with heteromultivalent paramagnetic liposomes for combined MR imaging and treatment of angiogenesis Nano Lett. 2010;10(1):52-58.
64. Kluza E, Jacobs I, Hectors SJ, et al. Dual-targeting of $\alpha v \beta 3$ and galectin-1 improves the specificity of paramagnetic/fluorescent liposomes to tumor endothelium in vivo. J Control Release. 2012;158(2):207-214.

65. Kiessling F, Huppert J, Zhang C, et al. RGD-labeled USPIO inhibits adhesion and endocytotic activity of alpha $\mathrm{v}$ beta3-integrin-expressing glioma cells and only accumulates in the vascular tumor compartment. Radiology. 2009;253(2):462-469.

66. Kircher MF, Willmann JK. Molecular body imaging: MR imaging, CT, and US. part I. principles. Radiology. 2012;263(3):633-643.

67. Kircher MF, Willmann JK. Molecular body imaging: MR imaging, CT, and US. Part II. Applications. Radiology. 2012;264(2):349-368.

68. Judenhofer MS, Wehrl HF, Newport DF, et al. Simultaneous PET-MRI: a new approach for functional and morphological imaging. Nat Med. 2008;14(4):459-465.

69. Wehrl HF, Sauter AW, Judenhofer MS, Pichler BJ. Combined PET/ MR imaging - technology and applications. Technol Cancer Res Treat. 2010;9(1):5-20.

70. Drzezga A, Souvatzoglou M, Eiber M, et al. First clinical experience with integrated whole-body PET/MR: comparison to $\mathrm{PET} / \mathrm{CT}$ in patients with oncologic diagnoses. J Nucl Med. 2012;53(6):845-855.

71. Weissleder R, Pittet MJ. Imaging in the era of molecular oncology. Nature. 2008;452(7187):580-589
International Journal of Nanomedicine

\section{Publish your work in this journal}

The International Journal of Nanomedicine is an international, peerreviewed journal focusing on the application of nanotechnology in diagnostics, therapeutics, and drug delivery systems throughou the biomedical field. This journal is indexed on PubMed Central,

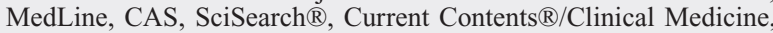

\section{Dovepress}

Journal Citation Reports/Science Edition, EMBase, Scopus and the Elsevier Bibliographic databases. The manuscript management system is completely online and includes a very quick and fair peer-review system, which is all easy to use. Visit http://www.dovepress.com/ testimonials.php to read real quotes from published authors. 